\title{
Static Two-Body Potential at Fifth Post-Newtonian Order
}

\author{
Stefano Foffa, ${ }^{1}$ Pierpaolo Mastrolia, ${ }^{2}$ Riccardo Sturani, ${ }^{3}$ Christian Sturm, ${ }^{4}$ and William J. Torres Bobadilla ${ }^{5}$ \\ ${ }^{1}$ Département de Physique Théorique and Centre for Astroparticle Physics, Université de Genève, CH-1211 Geneva, Switzerland \\ ${ }^{2}$ Dipartimento di Fisica ed Astronomia, Università di Padova, Via Marzolo 8, 35131 Padova, Italy \\ INFN, Sezione di Padova, Via Marzolo 8, 35131 Padova, Italy \\ ${ }^{3}$ International Institute of Physics (IIP), Universidade Federal do Rio Grande do Norte (UFRN) CP 1613, \\ 59078-970 Natal-RN Brazil \\ ${ }^{4}$ Universität Würzburg, Institut für Theoretische Physik und Astrophysik, Emil-Hilb-Weg 22, D-97074 Würzburg, Germany \\ ${ }^{5}$ Instituto de Física Corpuscular, Universitat de València-Consejo Superior de Investigaciones Científicas,
} Parc Científic, E-46980 Paterna, Valencia, Spain

(Received 5 March 2019; published 20 June 2019)

\begin{abstract}
We determine the gravitational interaction between two compact bodies up to the sixth power in Newton's constant, $G_{N}$, in the static limit. This result is achieved within the effective field theory approach to general relativity, and exploits a manifest factorization property of static diagrams which allows us to derive static post Newtonian (PN) contributions of $(2 n+1)$ order in terms of lower order ones. We recompute in this fashion the 1PN and 3PN static potential, and present the novel 5PN contribution.
\end{abstract}

DOI: 10.1103/PhysRevLett.122.241605

Introduction.-The study of the conservative dynamics of the two-body problem in general relativity (GR) is one of the pillars which allows us to determine the gravitational waveform templates for the LIGO/Virgo [1,2] data analysis pipeline $[3,4]$. The future generation of detectors such as the Einstein Telescope [5] and LISA [6] are expected to gain in sensitivity at least one order of magnitude with respect to the current generation of ground-based interferometers. Therefore, more accurate predictions on the theoretical side will be required for gravitational wave $(\mathrm{GW})$ astrophysics in the next decade $[7,8]$.

Deviations from the Newton potential due to GR effects can be studied in the so-called post-Newtonian (PN) framework, that is by expanding in powers of the two virial-related quantities, such as the compactness $R_{S} / r \sim$ $G_{N} m / r$ and the relative (squared) velocity $v^{2} \sim G_{N} m / r$, where $R_{S}, m, r$, and $G_{N}$ are the Schwarzschild radius of the system, its mass and size, and Newton's constant, respectively. The first complete 1PN computation was preformed by Einstein, Infeld, and Hoffmann in Ref. [9]; since then, the evaluation of the higher-order terms has been a formidable effort, whose current state of the art, after the calculation of the 2PN [10,11] and 3PN [12-14] contributions, is represented by the determination of the energy at 4PN order, which was achieved for the first time in

Published by the American Physical Society under the terms of the Creative Commons Attribution 4.0 International license. Further distribution of this work must maintain attribution to the author(s) and the published article's title, journal citation, and DOI.
Refs. [15-17] and later confirmed in Refs. [18-22] and in Refs. [23-26].

The next complexity level, namely the fifth postNewtonian approximation (5PN), is qualitatively important because of the first appearance of spin-independent finite size corrections. Several partial results towards this challenging precision level have become recently available in the so-called post-Minkowskian expansion, i.e., the expansion in $G_{N}$ only, for any given order in $v$, up to the third order in Newton's constant $G_{N}$ [27-32].

In the present Letter, we provide a novel contribution to the 5PN dynamics by tackling the determination of the highest possible power in $G_{N}$, namely $G_{N}^{6}$ at $5 \mathrm{PN}$, which amounts to determine the potential in the static limit. This goal is achieved by building on the ideas and the method of Ref. [24], where we computed the static potential at 4PN by adopting the effective field theory (EFT) approach to GR [33-37], in combination with techniques for the evaluation of multiloop scattering amplitudes in momentum space (see also Ref. [38] for a related computation in direct space). The computation of 5PN static corrections turns out to be actually less demanding than the corresponding 4PN ones, owing to a factorization property of the static contributions, yielding a drastic simplification at $o d d-\mathrm{PN}$ orders, which is explicit and intuitive in the EFT approach, and is formalized in the current Letter.

The $G_{N}^{6}$ subsector computed here is the highest order ever computed in powers of $G_{N}$ and, if done by brute force, involves the evaluation of the most complex integrals present at 5PN, which, within the EFT expansion, come from the graphs with the largest loop number. Therefore, its determination, presented here for the first time, paves the 
way to the completion of 5PN corrections, and confirms that the adopted methods are suitable to systematically tackle higher PN-order computations.

Effective field theory approach.-The evaluation of postNewtonian corrections to the dynamics of binary systems can be addressed within the by now established EFT framework [33], reviewed in Refs. [35,37,39]. Following the lines and notation of Refs. [23,40], we consider the action of the system, given by

$$
S=S_{\text {pp }}+S_{\text {bulk }}
$$

in terms of the world-line point particle action, representing the binary components (for spinless point masses and neglecting tidal effects)

$$
\begin{aligned}
S_{\mathrm{pp}} & =-\sum_{i=1,2} m_{i} \int d \tau_{i} \\
& =-\sum_{i=1,2} m_{i} \int \sqrt{-g_{\mu \nu}\left(x_{i}\right) d x_{i}^{\mu} d x_{i}^{\nu}},
\end{aligned}
$$

and of the canonical Einstein-Hilbert action plus a gaugefixing (harmonic condition) term $[18,41]$,

$$
\begin{aligned}
S_{\mathrm{bulk}}= & S_{\mathrm{EH}}+S_{\mathrm{GF}} \\
& =2 \Lambda^{2} \int d^{d+1} x \sqrt{-g}\left[R(g)-\frac{1}{2} \Gamma_{\mu} \Gamma^{\mu}\right],
\end{aligned}
$$

where $\Gamma^{\mu} \equiv g^{\rho \sigma} \Gamma_{\rho \sigma}^{\mu}$. In the above formula, $\Lambda^{-2} \equiv 32 \pi G_{N} L^{d-3}$, where $G_{N}$ is the three-dimensional Newton constant, and $L$ is an arbitrary length scale that keeps the correct dimensions of $\Lambda$ in dimensional regularization, which cancels out in the expression of physical observables. In this framework, a Kaluza-Klein (KK) parametrization of the metric [42-44] is usually adopted:

$$
g_{\mu \nu}=e^{2 \phi / \Lambda}\left(\begin{array}{cc}
-1 & A_{j} / \Lambda \\
A_{i} / \Lambda & e^{-c_{d} \phi / \Lambda} \gamma_{i j}-A_{i} A_{j} / \Lambda^{2}
\end{array}\right),
$$

with $\gamma_{i j} \equiv \delta_{i j}+\sigma_{i j} / \Lambda, c_{d}=2(d-1) /(d-2)$ and $i, j$ running over the $d$ spatial dimensions. Accordingly, the degrees of freedom of the graviton field are reparametrized in terms of a scalar field $\phi$, a vector field $A_{i}$, and a symmetric tensor field $\sigma_{i j}$. The field $A_{i}$ is not actually needed in the static limit because it always comes in association with the velocity of one of the compact bodies, so it will henceforth be set to zero.

In terms of the metric parametrization [Eq. (4)], with $A_{i}=0$, each world-line coupling to the gravitational degrees of freedom $\phi, \sigma_{i j}$ reads

$$
\begin{aligned}
& S_{p p}=-m \int d t e^{\phi / \Lambda} \sqrt{1-e^{-c_{d} \phi / \Lambda}\left(v^{2}+\frac{\sigma_{i j}}{\Lambda} v^{i} v^{j}\right)} \\
& \underset{\text { static }}{\longrightarrow}-m \int d t e^{\phi / \Lambda},
\end{aligned}
$$

and its Taylor expansion provides the various particlegravity vertices involving $\phi$, like the coupling of $\phi$ to matter fields, i.e., the $m \phi^{n}$ vertex,

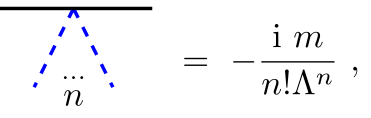

where black lines stands for matter and dashed blue lines indicate $\phi$ modes. Also the pure gravity sector $S_{\text {bulk }}$ can be explicitly written in terms of the KK variables; for the purpose of this Letter, it is sufficient to report here only the structure of the static terms not containing the field $\vec{A}$ [45]:

$$
S_{\text {bulk }} \supset \int d^{d+1} x \sqrt{\gamma}\left\{f\left(\sigma_{i j}\right)-c_{d}(\vec{\nabla} \phi)^{2}\right\},
$$

where $f$ is a function depending on the field $\sigma_{i j}$ only. The complete set of Feynman rules, also involving the fields $\sigma_{i j}$ and $A_{i}$ (respectively indicated by green and red lines), can be found in Ref. [24].

The two-body effective action can be found by integrating out the gravity fields from the above-derived actions

$$
\exp \left[i S_{\mathrm{eff}}\right]=\int D \phi D \sigma_{i j} \exp \left[i\left(S_{\mathrm{bulk}}+S_{\mathrm{pp}}\right)\right]
$$

Within the field-theoretical approach, the functional integration can be perturbatively expanded in terms of Feynman diagrams involving the gravitational degrees of freedom as internal lines only, viewed as dynamical fields emitted and absorbed by the point particles, which are taken as nondynamical sources. Each diagram shows a manifest power counting both in the bodies' relative velocities and in $G_{N}$ [any bulk vertex involving $k$ fields carries a factor $\left(G_{N}\right)^{(k / 2)-1}$ and any $m \phi^{n}$ vertex carries a factor $\left(G_{N}\right)^{(n / 2)}$, thus allowing for a systematic PN classification. The most elementary diagram in the EFT approach is represented by the Newton-potential graph

$$
\mathcal{V}_{N}=\underset{\vdots}{i}=-\frac{G_{N} m_{1} m_{2}}{r}
$$

naïvely dubbed as 0PN diagram.

Factorization theorem.-Definition: Static EFT-gravity diagrams can be classified according to the type of couplings between matter and $\phi$ fields. We can distinguish between factorizable graphs, which contain at least one $m \phi^{n}$ vertex with $n>1$, and prime graphs, which contain only matter- $\phi$ vertices of the type $m \phi$, namely where each $\phi$, coming from the bulk (and not propagating between bulk vertices), couples individually to matter (see Fig. 1, left).

Factorizable graphs can be obtained by sewing together two, or more, subgraphs that, upon merging, share a $m \phi^{n}$ vertex $(n>1)$ (see Fig. 1, right). 


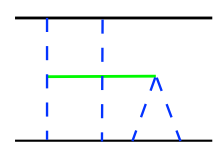

\begin{tabular}{|c|c|}
\hline$T$ & $T$ \\
\hline II & I \\
\hline 11 & $1 n$ \\
\hline 11 & 111 \\
\hline 11 & 11 \\
\hline
\end{tabular}

FIG. 1. Examples of a prime 4PN graph (left) and of a factorizable 5PN graph (right): the latter can be obtained by sewing the former and the Newton potential diagram.

Proposition: Inspection of Eq. (7) shows that the only bulk gravity vertices allowed in a static graph are those containing (1) zero or two $\phi \mathrm{s}$ and (2) any number of $\sigma_{i j} \mathrm{~s}$; the latter cannot however be attached to any particle, see Eq. (5), so they can just propagate between bulk vertices. This observation is crucial to prove an important property of prime graphs, which constitute the first novel result of this communication.

Theorem: Static prime graphs exist only at even $2 n$-PN orders. Equivalently, static graphs at odd $(2 n+1) \mathrm{PN}$ orders are factorizable.

Proof: This statement can be proven by showing that any prime static graph must have an even number of $\phi$ fields attached to the particles.

For the Newtonian graph, it is trivially true by construction. Graphs generated by PN corrections, $\mathcal{O}\left(G_{N}^{2}\right)$, necessarily contain bulk vertices $\phi \phi \sigma^{k}$ (with $k \geq 1$ ), coming from the expansions of the graviton self-interaction terms. For these diagrams, two cases may occur: (1) each internal $\phi$ propagator is contracted on the one side with a matter- $\phi$ vertex, and, on the other side, with a $\phi \phi \sigma^{k}$ vertex; therefore it contributes with one power of $m_{i}$ to the mass dimensions of the graph. (2) A $\phi$ propagator, not coupled with matter, must necessarily connect two $\phi \phi \sigma^{k}$ vertices; therefore it does not contribute to the mass dimensions of the graph. Since the bulk vertices between $\phi$ and $\sigma$ fields $(\phi \phi \sigma, \phi \phi \sigma \sigma, \ldots)$ are quadratic in $\phi$, and because prime graphs are characterized by either (1) or (2), we can conclude that the total number of $\phi$ fields that depart from the bulk vertices and couple to matter (either $m_{1}$ or $m_{2}$ ) is an even number.

This implies that, being $n_{i}$, the number of $\phi$ fields coupled to the matter $m_{i}(i=1,2)$, the total masslike power of static prime graphs is $m_{1}^{n_{1}} m_{2}^{n_{2}}$, with $n_{1}+n_{2}=2 n$ and $n \in \mathbb{N}^{+}$. On the other side, they correspond to static classical contributions; therefore, they must consequently scale as $G_{N}^{(2 n-1)} m_{1}^{n_{1}} m_{2}^{n_{2}} / r^{(2 n-1)}$ (classical diagrams do not contain loops in the dynamical fields), finally implying that they belong to an even-PN order.

Due to the factorization theorem, the general structure of the contribution to the potential of a given $n \mathrm{PN}$ factorizable diagram, in terms of the product of lower PN-order graphs, reads

$$
\mathcal{V}_{n}^{\text {factorizable }}=\left(\mathcal{V}_{L, n_{1}} \times \mathcal{V}_{R, n_{2}}\right) \times \mathcal{K} \times \mathcal{C},
$$

where (1) the PN orders, $n_{1}$ of the left graph $\mathcal{V}_{L}$ and $n_{2}$ of the right graph $\mathcal{V}_{R}$, are such that $n_{1}+n_{2}+1=n$,
(2) $\mathcal{K}$ accounts for the new matter- $\phi^{k}$ vertex of $\mathcal{V}_{n}$ (emerging from the sewing) out of the ones included in the lower order contributions, $\mathcal{V}_{L, n_{1}}$ and $\mathcal{V}_{R, n_{2}}$, and (3) $\mathcal{C}=C_{n}^{\text {factorizable }} /\left(C_{L, n_{1}} \times C_{R, n_{2}}\right)$ where the $C$ s are the combinatoric factors associated with each graph.

Gravity and field theory diagrams.-In a quantum field theory approach, any EFT-gravity graph can be interpreted as four-particle scattering amplitude [24]. The contribution of each amplitude to the two-body potential $\mathcal{V}$ can be obtained by taking its Fourier transform,

$$
\mathcal{V}=\mathrm{i} \lim _{d \rightarrow 3} \int_{p} e^{\mathrm{i} p \cdot r}{ }^{2}{ }^{3}
$$

where, $\int_{p} \equiv \int d^{d} p /(2 \pi)^{d}$, the box diagram stands for a generic EFT-gravity diagram, and $p$ is the momentum transfer of the source (assuming momentum conservation $p_{1}+p_{2}=p_{3}+p_{4}$, then $\left.p=p_{3}-p_{2}=p_{1}-p_{4}\right)$. Since the sources, represented by black lines, are static and do not propagate, any EFT-gravity amplitude at order $G_{N}^{\ell}$ can be mapped into an $(\ell-1)$ loop two-point function with massless internal lines and external momentum $p$ $\left(p^{2} \neq 0\right)$ [24]. This observation was crucial to perform the 4PN static calculation by employing computational techniques developed for the evaluation of multiloop Feynman integrals in high-energy particle physics. Moreover, in the current Letter, we observe that the integration on $p$ can be seen as an additional loop integration; hence it can be represented by an $\ell$-loop vacuum diagram, obtained by joining the external legs into a propagatorlike line (indicated by an inner black line), as

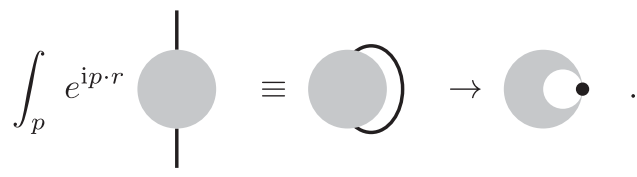

In the last step, we introduce a suggestive diagrammatic representation of the Fourier integral as an $\ell$-loop vacuum graph by pinching the internal black line. The presence of the dot " $\bullet "$ indicates the residual $r$ dependence of the contribution (not to be confused by fully massless, hence scaleless vacuum diagrams that vanish in dimensional regularization).

In the case of factorizable EFT-diagrams, the pinching generates the product of factorized vacuum diagrams. For example, the contribution to the 5PN potential of the diagram in Fig. 1 (right) becomes

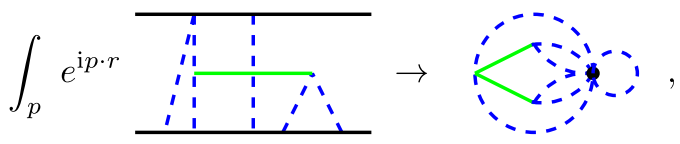


directly representing the product of the Newton potential and of a 4PN term, respectively, represented by a one-loop and a five-loop vacuum diagram.

Results.-We verified that the static potential at 1PN and 3PN [40] can be derived by applying the factorization theorem to the relevant diagrams.

We now apply the factorization theorem to the 5PN case, computed for the first time in the present Letter. There are 154 diagrams to evaluate and it is convenient to divide them in four classes, according to their factorization patterns. For ease of notation, we arrange the 5PN static graphs in four subsets, displaying the lower-PN corrections they stem out from, and give the factors $\mathcal{K}$ and $\mathcal{C}$ as understood. (1) There are 11 diagrams composed of six Newtonian factors, combined in different ways, and schematically represented as

$$
\left(\begin{array}{l}
1 \\
1 \\
1
\end{array}\right)^{6} .
$$

The contribution to the $5 \mathrm{PN}$ potential coming from this set of diagrams is

$$
\begin{aligned}
\mathcal{V}_{N^{6}}= & \frac{1}{720} \frac{G_{N}^{6} m_{1}^{6} m_{2}}{r^{6}}+\frac{1}{3} \frac{G_{N}^{6} m_{1}^{5} m_{2}^{2}}{r^{6}} \\
& +3 \frac{G_{N}^{6} m_{1}^{4} m_{2}^{3}}{r^{6}}+\left(m_{1} \leftrightarrow m_{2}\right) .
\end{aligned}
$$

(2) One can build static factorizable diagrams as products of three Newtonian graphs, and either of the 2PN prime graphs, schematically represented as:

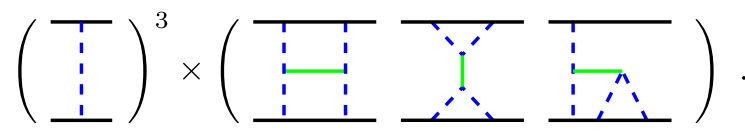

This set contains 49 diagrams, 9 of which are vanishing, because one of the 2PN factors is indeed zero. The combined contribution of the remaining diagrams is

$$
\begin{aligned}
\mathcal{V}_{N^{3} \times 2 \mathrm{PN}}= & \frac{1}{18} \frac{G_{N}^{6} m_{1}^{6} m_{2}}{r^{6}}+\frac{16}{3} \frac{G_{N}^{6} m_{1}^{5} m_{2}^{2}}{r^{6}} \\
& +\frac{229}{6} \frac{G_{N}^{6} m_{1}^{4} m_{2}^{3}}{r^{6}}+\left(m_{1} \leftrightarrow m_{2}\right) .
\end{aligned}
$$

(3) In this class, we consider 5PN diagrams schematically represented by the product of one Newtonian graph with each of the 25 static prime 4PN diagrams studied in Ref. [24] (the cardinal number attached to each graph is the same as in Ref. [24], for ease of comparison)

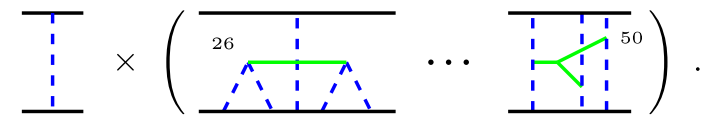

This set contains 79 diagrams, 16 of which are vanishing (due to vanishing $4 \mathrm{PN}$ factors). The remaining 63 diagrams give

$$
\begin{aligned}
\mathcal{V}_{N \times 4 \mathrm{PN}}= & \frac{1}{5} \frac{G_{N}^{6} m_{1}^{6} m_{2}}{r^{6}}+\frac{23}{3} \frac{G_{N}^{6} m_{1}^{5} m_{2}^{2}}{r^{6}} \\
& +\frac{166}{3} \frac{G_{N}^{6} m_{1}^{4} m_{2}^{3}}{r^{6}}+\left(m_{1} \leftrightarrow m_{2}\right) .
\end{aligned}
$$

Interestingly, let us observe that although this set contains contributions that are individually divergent in the $d \rightarrow 3$ limit, as well as factors of $\pi^{2}$, within their sum all poles and irrational factors cancel, and the result is indeed finite and rational.

(4) Finally, we consider static 5PN diagram formed by the product of two $2 \mathrm{PN}$ graphs, schematically represented as

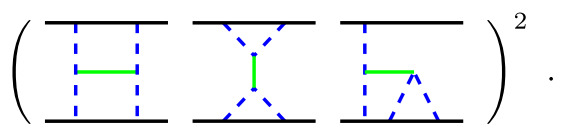

This term contains 15 5PN graphs, 5 of which are manifestly vanishing, while the contribution of the remaining 10 diagrams reads:

$$
\begin{aligned}
\mathcal{V}_{(2 \mathrm{PN})^{2}}= & \frac{1}{18} \frac{G_{N}^{6} m_{1}^{6} m_{2}}{r^{6}}+\frac{11}{6} \frac{G_{N}^{6} m_{1}^{5} m_{2}^{2}}{r^{6}} \\
& +\frac{37}{3} \frac{G_{N}^{6} m_{1}^{4} m_{2}^{3}}{r^{6}}+\left(m_{1} \leftrightarrow m_{2}\right) .
\end{aligned}
$$

Total 5PN static potential: by combining all the previous results, the expression for the static sector of the 5PN potential finally reads

$$
\begin{aligned}
\mathcal{V}_{\text {static }}^{(5 \mathrm{PN})}= & \mathcal{V}_{N^{6}}+\mathcal{V}_{N^{3} \times 2 \mathrm{PN}}+\mathcal{V}_{N \times 4 \mathrm{PN}}+\mathcal{V}_{(2 \mathrm{PN})^{2}} \\
= & \frac{5}{16} \frac{G_{N}^{6} m_{1}^{6} m_{2}}{r^{6}}+\frac{91}{6} \frac{G_{N}^{6} m_{1}^{5} m_{2}^{2}}{r^{6}} \\
& +\frac{653}{6} \frac{G_{N}^{6} m_{1}^{4} m_{2}^{3}}{r^{6}}+\left(m_{1} \leftrightarrow m_{2}\right) .
\end{aligned}
$$

This expression contains the genuine $G_{N}^{6}$ contribution coming from graphs, without contributions generated from lower- $G_{N}$ terms when using the equations of motion to eliminate terms that, at least, have a quadratic dependence on the acceleration. Together with the factorization theorem, the above expressions constitute the second important result of this Letter [46].

Check of test particle limit: it is possible to verify that the coefficient of the term $m_{1}^{6} m_{2}$ agrees with what can be expected from the extreme mass ratio limit $m_{2} \ll m_{1}$. In this limit, where only the graphs displayed in Fig. 2 contribute, it is possible to consider the body with mass $m_{2}$ as a test particle in the Schwarzschild metric generated by the body with mass $m_{1}$.

The action describing the dynamics of the test body has still the form $S_{\text {pp }}$ described in Eq. (2), but with $g_{\mu \nu}$ given by the Schwarzschild metric in harmonic coordinates (which is 


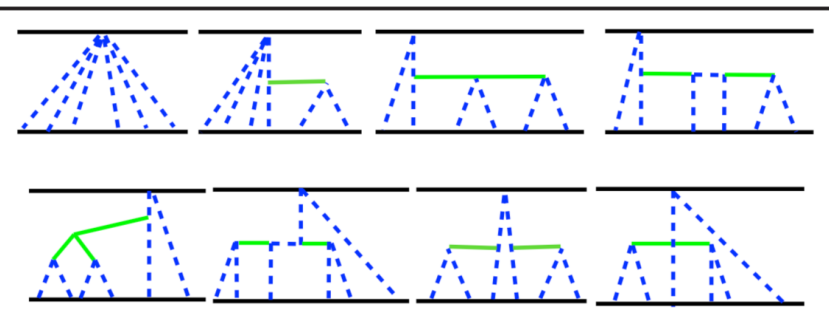

FIG. 2. 5PN graphs contributing to the test-particle limit. The last graph (bottom right) does not contribute to the 5PN potential, because its 4PN subdiagram vanishes.

obtained from the traditional form by the simple radial coordinate shift $r \rightarrow r+G_{N} m_{1}$ ) instead of the Minkowski one.

In the static limit, $v_{2}=0$, only the term $g_{00}$ survives, and the effective Lagrangian reads

$$
\mathcal{L}_{\text {static }}^{m_{2} \ll m_{1}}=-m_{2} \sqrt{-g_{00}}=-m_{2} \sqrt{\frac{1-\frac{G_{N} m_{1}}{r}}{1+\frac{G_{N} m_{1}}{r}}} .
$$

By expanding this expression in $G_{N} m_{1} / r$, one obtains the sequence $\left(1,-\frac{1}{2}, \frac{1}{2},-\frac{3}{8}, \frac{3}{8},-\frac{5}{16}, \frac{5}{16},-\frac{35}{128}, \frac{35}{128},-\frac{63}{256}, \ldots\right)$ of all the coefficients of the $n \mathrm{PN}$ static terms $G_{N}^{n} m_{1}^{n} m_{2} / r^{n}$, including the $-\frac{5}{16}$ of the 5PN term reported in Eq. (22) (where the potential is correctly reported with opposite sign with respect to the Lagrangian term).

Conclusion.-We studied the two-body conservative dynamics at fifth post-Newtonian order (5PN) in the static limit within the EFT approach to general relativity. We determined an essential contribution of the complete 5PN potential at $\mathcal{O}\left(G_{N}^{6}\right)$, coming from 154 Feynman diagrams. We proved a factorization property of the static diagrams at odd-PN order, and exploited it to show that their contribution can be determined recursively, from lower PN orders. The result of the static potential at order $G_{N}^{6}$ is found to be finite and rational-a property clearly inherited from the static $G_{N}^{5}$ sector-and exhibits the expected Schwarzschild-like behavior in the extreme mass ratio limit. The factorization theorem can be applied as well to even-PN orders, where it simplifies the evaluation of a large subset of the contributing diagrams, therefore becoming a powerful tool to systematize and to ease the computations at high-PN orders.

S. F. is supported by the Fonds National Suisse and by the SwissMap NCCR. P. M. is supported by the Supporting TAlent in ReSearch at Padova University (UniPD STARS Grant "Diagrammalgebra"). R. S. is partially supported by CNPq. W. J. T. has been supported in part by Grants No. FPA2017-84445-P and No. SEV-20140398 (AEI/ERDF, EU), the COST Action CA16201 PARTICLEFACE, and the "Juan de la Cierva Formación" program (FJCI-2017-32128).
[1] J. Aasi et al. (LIGO Scientific Collaboration), Classical Quantum Gravity 32, 074001 (2015).

[2] F. Acernese et al. (VIRGO Collaboration), Classical Quantum Gravity 32, 024001 (2015).

[3] A. Taracchini, Y. Pan, A. Buonanno, E. Barausse, M. Boyle, T. Chu, G. Lovelace, H. P. Pfeiffer, and M. A. Scheel, Phys. Rev. D 86, 024011 (2012).

[4] P. Schmidt, F. Ohme, and M. Hannam, Phys. Rev. D 91, 024043 (2015).

[5] M. Punturo et al., Classical Quantum Gravity 27, 194002 (2010).

[6] H. Audley et al. (LISA Collaboration), arXiv:1702.00786.

[7] L. Lindblom, B. J. Owen, and D. A. Brown, Phys. Rev. D 78, 124020 (2008).

[8] A. Antonelli, A. Buonanno, J. Steinhoff, M. van de Meent, and J. Vines, Phys. Rev. D 99, 104004 (2019).

[9] A. Einstein, L. Infeld, and B. Hoffmann, Ann. Math. 39, 65 (1938).

[10] T. Damour, in Les Houches Summer School on Gravitational Radiation Les Houches, France, 1982 (Elsevier Science Ltd, Amsterdam, 1982).

[11] T. Damour and G. Schäfer, Gen. Relativ. Gravit. 17, 879 (1985).

[12] T. Damour, P. Jaranowski, and G. Schäfer, Phys. Lett. B 513, 147 (2001).

[13] L. Blanchet, T. Damour, and G. Esposito-Farese, Phys. Rev. D 69, 124007 (2004).

[14] Y. Itoh and T. Futamase, Phys. Rev. D 68, 121501(R) (2003).

[15] T. Damour, P. Jaranowski, and G. Schäfer, Phys. Rev. D 89, 064058 (2014).

[16] T. Damour, P. Jaranowski, and G. Schäfer, Phys. Rev. D 91, 084024 (2015).

[17] T. Damour, P. Jaranowski, and G. Schäfer, Phys. Rev. D 93, 084014 (2016).

[18] L. Bernard, L. Blanchet, A. Bohé, G. Faye, and S. Marsat, Phys. Rev. D 93, 084037 (2016).

[19] L. Bernard, L. Blanchet, A. Bohé, G. Faye, and S. Marsat, Phys. Rev. D 95, 044026 (2017).

[20] L. Bernard, L. Blanchet, A. Bohé, G. Faye, and S. Marsat, Phys. Rev. D 96, 104043 (2017).

[21] T. Marchand, L. Bernard, L. Blanchet, and G. Faye, Phys. Rev. D 97, 044023 (2018).

[22] L. Bernard, L. Blanchet, G. Faye, and T. Marchand, Phys. Rev. D 97, 044037 (2018).

[23] S. Foffa and R. Sturani, Phys. Rev. D 87, 064011 (2013).

[24] S. Foffa, P. Mastrolia, R. Sturani, and C. Sturm, Phys. Rev. D 95, 104009 (2017).

[25] S. Foffa and R. Sturani, arXiv:1903.05113.

[26] S. Foffa, R. A. Porto, I. Rothstein, and R. Sturani, arXiv:1903.05118.

[27] T. Ledvinka, G. Schäfer, and J. Bicak, Phys. Rev. Lett. 100, 251101 (2008).

[28] S. Foffa, Phys. Rev. D 89, 024019 (2014).

[29] L. Blanchet and A. S. Fokas, Phys. Rev. D 98, 084005 (2018).

[30] T. Damour, Phys. Rev. D 97, 044038 (2018).

[31] C. Cheung, I. Z. Rothstein, and M. P. Solon, Phys. Rev. Lett. 121, 251101 (2018). 
[32] Z. Bern, C. Cheung, R. Roiban, C.-H. Shen, M. P. Solon, and M. Zeng, Phys. Rev. Lett. 122, 201603 (2019).

[33] W. D. Goldberger and I. Z. Rothstein, Phys. Rev. D 73, 104029 (2006).

[34] W. D. Goldberger, in Les Houches Summer School-Session 86: Particle Physics and Cosmology: The Fabric of Spacetime Les Houches, France, 2006 (Elsevier Science Ltd, Amsterdam, 2007).

[35] S. Foffa and R. Sturani, Classical Quantum Gravity 31, 043001 (2014).

[36] I. Z. Rothstein, Gen. Relativ. Gravit. 46, 1726 (2014).

[37] R. A. Porto, Phys. Rep. 633, 1 (2016).

[38] T. Damour and P. Jaranowski, Phys. Rev. D 95, 084005 (2017).
[39] M. Levi, arXiv:1807.01699.

[40] S. Foffa and R. Sturani, Phys. Rev. D 84, 044031 (2011).

[41] L. Blanchet, Living Rev. Relativity 17, 2 (2014).

[42] L. Blanchet and T. Damour, Ann. Inst. H. Poincare Phys. Theor. 50, 377 (1989).

[43] B. Kol and M. Smolkin, Classical Quantum Gravity 25, 145011 (2008).

[44] B. Kol and M. Smolkin, Phys. Rev. D 77, 064033 (2008).

[45] It is understood that spatial indices in this expression are contracted by means of the spatial metric $\gamma_{i j}$, which implies the appearance of extra $\sigma$ fields.

[46] Eq. (22) has been later confirmed in [47].

[47] J. Blümlein, A. Maier, and P. Marquard, arXiv:1902.11180. 